\title{
Trace identities and their semiclassical implications
}

\author{
Uzy Smilansky $\ddagger$ \\ Fachbereich Physik, Philipps-Universitaet Marburg, D-35032 Marburg, Germany
}

\begin{abstract}
The compatibility of the semiclassical quantization of area-preserving maps with some exact identities which follow from the unitarity of the quantum evolution operator is discussed. The quantum identities involve relations between traces of powers of the evolution operator. For classically integrable maps, the semiclassical approximation is shown to be compatible with the trace identities. This is done by the identification of stationary phase manifolds which give the main contributions to the result. The same technique is not applicable for chaotic maps, and the compatibility of the semiclassical theory in this case remains unsettled. The compatibility of the semiclassical quantization with the trace identities demonstrates the crucial importance of non-diagonal contributions.
\end{abstract}

PACS numbers: 05.45.+b, 03.65.Sq

\section{Introduction}

This paper focuses on quantum maps which are represented by unitary evolution operators on a Hilbert space of a finite dimension. The quantum map propagates any initial state in the Hilbert space by

$$
\psi_{t+1}=U \psi_{t}=U^{t+1} \psi_{0} .
$$

It is assumed that there exists an underlying area preserving classical map which acts on a compact phase space $\mathcal{M}$, which can be considered as the counterpart of the quantum map within the semiclassical approximation. That is,

(i) The semiclassical quantization of the classical map (see section 3) provides an approximation to the exact quantum map. The semiclassical approximant is unitary $U_{\mathrm{scl}}\left(U_{\mathrm{scl}}\right)^{\dagger}=I$ and satisfies the composition rule $U_{\mathrm{scl}}^{t+s}=U_{\mathrm{scl}}^{t} U_{\mathrm{scl}}^{s}$ within the accuracy margin of the semiclassical approximation.

(ii) The dimension of the Hilbert space, $M$, is related to the classical phase space volume $|\mathcal{M}|$ by

$$
M=\left[\frac{|\mathcal{M}|}{(2 \pi \hbar)^{f}}\right]
$$

where [.] stands for the integer part, and $f$ is the number of classical freedoms.

$\ddagger$ Permanent address: Department of Physics of Complex Systems, The Weizmann Institute of Science, Rehovot 76100, Israel 
Consider a quantum map $U$ and introduce the notation $t_{n} \equiv \operatorname{tr} U^{n}$. The fact that $U$ is unitary imposes various relations amongst the $t_{n}$ which should be satisfied identically. The purpose of this paper is to study to what extent the semiclassical approximation is compatible with a certain class of identities, which, to the best of my knowledge, was not examined in this context till now. To give an idea, the most simple version of the identities to be considered is

$$
\lim _{\epsilon \rightarrow 0} \epsilon \sum_{n=n_{0}}^{\infty} t_{n}^{*} t_{n+\nu} \mathrm{e}^{-n \epsilon}=t_{\nu}
$$

for arbitrary integers $n_{0}$ and $\nu$. These identities will be proved and discussed in detail in section 2 . However, before doing this, it is instructive to review a few other identities, which involve the $t_{n}$, and which were used in past investigations of the semiclassical approximation [1, 2, 3, 4, 4 .

The first class of identities can be derived by studying the properties of the characteristic polynomial

$$
p_{U}(z) \equiv \operatorname{det}(I-z U)=\sum_{m=0}^{M} a_{m} z^{m} .
$$

Since $p_{U}\left(U^{\dagger}\right)=0$,

$$
a_{0}=1=-\sum_{m=1}^{M} a_{m}\left(U^{\dagger}\right)^{m} .
$$

Multiplying by $U^{n}(n>M)$ and taking the trace one gets

$$
t_{n}=-\sum_{m=1}^{M} a_{m} t_{n-m} .
$$

By successive applications of the above relation, all the traces $t_{n}$ with $n>M$ can be expressed in terms of the traces of the $M$ lowest powers.

An important consequence of the unitarity of $U$ is the inversive symmetry of the coefficients $a_{m}$,

$$
a_{m}=e^{i \Theta} a_{M-m}^{*},
$$

where $\operatorname{det}(-U) \equiv e^{i \Theta}$. One can utilize the inversive symmetry to obtain identities between the $t_{n}$ by invoking Newton's identities. They relate the traces $t_{n}$ and the coefficients of the secular polynomial $a_{m}$ :

$$
a_{m}=-\frac{1}{m}\left(t_{m}+\sum_{k=1}^{m-1} a_{k} t_{m-k}\right) .
$$

Since $a_{m}=0$ for $m>M$, the $t_{n}$ for all $n>M$ depend linearly on the lower $m \leq M$ traces, which is consistent with our previous observation. Successive iterations yield explicit expressions for the coefficients $a_{m}$ in terms of the $t_{n}$, and one can substitute them in (5) or in (6).

The significance of such relations in the semiclassical context is due to the fact that the $t_{n}$ are expressed semiclassically as sums over $n$-periodic orbits of the classical map. Thus, the compatibility with the exact identities implies that there exist identities 
relating sums over periodic orbits of different periods which are satisfied within the margins of the semiclassical accuracy. The resulting identities between the $t_{n}$ are getting complicated as $n$ and $M$ increase, and therefore their compatibility with the semiclassical approximation was seldom tested [5].

Another set of identities which will be shown to be closely related to the present work were introduced by M.V. Berry in [6]. He considered the spectral density of a quantum hamiltonian

$$
d(E)=\sum_{n=1}^{\infty} \delta\left(E-E_{n}\right)
$$

and its smooth approximant

$$
d_{\epsilon}(E)=\sum_{n=1}^{\infty} \delta_{\epsilon}\left(E-E_{n}\right) \text { with } \delta_{\epsilon}(x)=\frac{1}{\pi} \frac{\epsilon}{\epsilon^{2}+x^{2}} .
$$

Assuming that the spectrum has no degeneracies $\left(E_{n} \neq E_{m}\right.$ when $\left.n \neq m\right)$, one finds,

$$
2 \pi \lim _{\epsilon \rightarrow 0} \epsilon d_{\epsilon}^{2}(E)=d(E) .
$$

Substituting the semiclassical trace formula in both sides of (10) one sees that the left hand side is quadratic while the right hand side is linear in the periodic orbit amplitudes. Integrating (10) over a sufficiently large energy domain, the contributions of long orbits to the right hand side can be made arbitrarily small, while their contribution to the left hand side will not be smoothed out. This observation led Berry to conclude that the long periodic orbits must contain information about the short periodic orbits if the semiclassical approximation is compatible with (10). This information is stored as correlations between actions of periodic orbits, because pairs of distinct periodic orbits combine together to give an amplitude of order $\epsilon^{-1}$ in $d_{\epsilon}^{2}(E)$, which, upon multiplying by $\epsilon$ reproduce the periodic orbit contributions to the oscillatory parts of $d(E)$. This is a highly "non-diagonal" effect, which needs very special correlations between the actions to get the correct result. This observation shows that the use of identities of this type comes naturally in the context of the study of classical action correlations and their effect on the statistics of the quantum spectra [9, 10, 11.

J. Keating [7] (see also [8) generalized (10) and used it in his studies of the spectrum of the Riemann zeros. The "non-diagonal" correlations which are necessary to prove the identities are introduced by using the Hardy-Littlewood conjecture on the correlations between primes.

E. Bogomolny 12 tested (10) for the spectrum of a rectangular billiard with periodic boundary conditions. By considering carefully the stationary phase manifolds in the sums over periodic orbits which arise from the left hand side, he was able to perform the summations and to demonstrate the compatibility of the semiclassical approximation with (10). The same methods were later used to analyze integrable systems in general. The present work was inspired by a seminar given by Professor Bogomolny, and it can be considered as an extension of Bogomolny's ideas to quantum maps and their semiclassical approximation.

This manuscript is arranged in the following way. The trace identities which will be the main tool in the present analysis will be derived in the next section. The semiclassical quantization of area preserving maps will be reviewed in section 3 and 
the compatibility of the semiclassical approximation will be demonstrated in section 1 for integrable maps. The close relation between the compatibility problem and the correlations between actions of periodic orbits will be addressed at the end of this section. The difficulties encountered in attempting to use the same methods to study the compatibility in the case of hyperbolic maps are discussed as well. By applying the trace identities to certain quantum graphs, it is possible to derive combinatorial identities which involve Krawtchouk polynomials 13, 14]. These polynomial are important building blocks in the theory of error correcting codes [15], and the identities might be of use in this branch of mathematics. Since this application is not directly connected to the issue of the semiclassical compatibility, it will be presented in the appendix.

\section{Trace identities}

Consider a unitary matrix $U$ of dimension $M$. Its spectrum consists of $M$ points on the unit circle $\left\{\mathrm{e}^{i \theta_{m}}, m=1, \cdots, M\right\}$, where the eigenphases are real and are assumed to be distinct. Recalling the notation $t_{n} \equiv \operatorname{tr} U^{n}$, the following identities hold for arbitrary integers $n_{0}$ and $\nu$ :

$$
\lim _{\epsilon \rightarrow 0} \epsilon \sum_{n=n_{0}}^{\infty} t_{n}^{*} t_{n+\nu} \mathrm{e}^{-n \epsilon}=t_{\nu}
$$

To prove these relations one substitutes $t_{n}=\sum_{m=1}^{M} \mathrm{e}^{i n \theta_{m}}$ and after summing the geometric series, one uses

$$
\lim _{\epsilon \rightarrow 0} \frac{\epsilon}{1-\mathrm{e}^{i\left(\theta_{m}-\theta_{m^{\prime}}\right)-\epsilon}}=\left\{\begin{array}{lll}
1 & \text { for } & \left(\theta_{m}-\theta_{m^{\prime}}\right)=0 \\
0 & \text { for } & \left(\theta_{m}-\theta_{m^{\prime}}\right) \neq 0
\end{array}\right\}=\delta_{m, m^{\prime}} .
$$

The condition that there are no degeneracies in the spectrum of $U$ is used to justify the rightmost equality in (12) .

A few points are worth noticing:

- The $\epsilon \rightarrow 0$ limit of the sum weighted by $\mathrm{e}^{-n \epsilon}$ can be interpreted as a time average

$$
\lim _{\epsilon \rightarrow 0} \epsilon \sum_{n=n_{0}}^{\infty}(\cdot)_{n} \mathrm{e}^{-n \epsilon}=\lim _{N \rightarrow \infty} \frac{1}{N} \sum_{n=n_{0}}^{n_{0}+N}(\cdot)_{n} .
$$

- For $\nu=0$, and using $t_{0}=M$, one gets

$$
\lim _{\epsilon \rightarrow 0} \epsilon \sum_{n=0}^{\infty} \frac{1}{M}\left|t_{n}\right|^{2} \mathrm{e}^{-n \epsilon}=1 .
$$

Thus, the time average of $\frac{1}{M}\left|t_{n}\right|^{2}$ approaches 1 , a result familiar from the study of the spectral form-factor for unitary matrices [16].

- The spectral density of $U$ on the unit circle can be written as

$$
d(\theta)=\sum_{l=1}^{M} \delta\left(\theta-\theta_{l}\right)=\lim _{\epsilon \rightarrow 0} d_{\epsilon}(\theta) ; d_{\epsilon}(\theta) \equiv \frac{1}{2 \pi} \sum_{m=-\infty}^{\infty} t_{m} \mathrm{e}^{-i m \theta-\epsilon|m|} .
$$

Using (11), one can easily derive

$$
2 \pi \lim _{\epsilon \rightarrow 0} \epsilon d_{\epsilon}^{2}(\theta)=d(\theta)
$$


which is the analogue of $(10)$ for the spectrum of unitary operators.

The following identities which involve products of more than two traces can be proven with the help of (12)

$$
\begin{aligned}
t_{\nu}= & \lim _{\epsilon_{1} \rightarrow 0} \cdots \lim _{\epsilon_{k} \rightarrow 0} \epsilon_{1} \cdots \epsilon_{k} \\
& \sum_{n_{1}=n_{1_{0}}}^{\infty} \cdots \sum_{n_{k}=n_{k_{0}}}^{\infty} t_{n_{1}}^{*} \cdots t_{n_{k}}^{*} t_{\left(n_{1}+\cdots+n_{k}\right)+\nu} \exp \left(-\sum_{i=1}^{k} n_{i} \epsilon_{i}\right),
\end{aligned}
$$

where the $n_{i_{0}}$ are arbitrary integers. The identities (17) can be also written as

$$
\begin{aligned}
t_{\nu_{1}+\cdots+\nu_{k}}= & \lim _{\epsilon_{1} \rightarrow 0} \cdots \lim _{\epsilon_{k} \rightarrow 0} \epsilon_{1} \cdots \epsilon_{k} \\
& \sum_{n_{1}=n_{1_{0}}}^{\infty} \cdots \sum_{n_{k}=n_{k_{0}}}^{\infty} t_{n_{1}+\cdots+n_{k}}^{*} t_{n_{1}+\nu_{1}} \cdots t_{n_{k}+\nu_{k}} \exp \left(-\sum_{i=1}^{k} n_{i} \epsilon_{i}\right) .
\end{aligned}
$$

The equivalence of (17) and (18) can be shown by taking the complex conjugate of (18), denoting $\nu_{1}+\cdots+\nu_{k}=-\nu$ and shifting the summation variables $n_{i}$ by $\nu_{i}$. The shifts do not affect the result since they can be absorbed in the arbitrary $\left\{n_{i_{0}}\right\}$.

The compatibility of the semiclassical approximation for $t_{n}$ with the identities $(11,17)$ will be investigated in section 4, after the semiclassical approximation for $t_{n}$ will be reviewed in the following section.

\section{Semiclassical quantization of maps}

The quantum unitary operator $U$ to be investigated, is assumed to be the analogue of an area preserving map $\mathcal{F}$ acting on a finite phase space domain $\mathcal{M}$ with area $|\mathcal{M}|$. (For the sake of simplicity the maps to be considered act on manifolds with $f=1$, and will be assumed to have the twist property. The semiclassical treatment can be extended to the general case.) The phase space coordinates are denoted by $\gamma=(q, p)$ and $\gamma$ is mapped to $\bar{\gamma} \equiv \mathcal{F}(\gamma)$. Area-preserving maps can be derived from a generating function (action) $\Phi(q, \bar{q})$

$$
p=-\frac{\partial \Phi(q, \bar{q})}{\partial q} \quad ; \quad \bar{p}=\frac{\partial \Phi(q, \bar{q})}{\partial \bar{q}} .
$$

The explicit mapping function $\bar{\gamma}=\mathcal{F}(\gamma)$ is obtained by solving the implicit relations (19). The twist condition ensures that the implicit equations (19) have a unique solution.

In the case the map is integrable, let $I$ be the invariant momentum (action variable) under the action of the map and $\phi$ the canonically conjugate angle variable. The domain of the map is the annulus $I \in\left[I_{\min }, I_{\max }\right], \phi \in[0,2 \pi)$, and $|\mathcal{M}|=$ $2 \pi\left(I_{\max }-I_{\min }\right)$. In this representation, the generating function must take the form $\Phi(\phi, \bar{\phi})=\Phi(\bar{\phi}-\phi)$. The explicit map is

$$
\bar{I}=I \quad ; \quad \Delta \phi=\bar{\phi}-\phi=f(I)
$$

Here, $f(I)$ (the angular velocity) is the inverse of the generating relation $I=\Phi^{\prime}(\Delta \phi)$, which gives $\Delta \phi$ in terms of $I$. The twist condition is fulfilled if $\Phi^{\prime \prime}(\Delta \phi) \neq 0$.

A classical trajectory is obtained by applying the map to an arbitrary initial point in $\mathcal{M}$, and the corresponding action is accumulated along the trajectory. 
The semiclassical quantization of $\mathcal{F}$ in the $q$ representation is [17, 3]:

$$
\langle q|U| \bar{q}\rangle_{\mathrm{scl}}=\left(\frac{1}{2 \pi \hbar i}\right)^{\frac{1}{2}}\left[\frac{\partial^{2} \Phi(q, \bar{q})}{\partial q \partial \bar{q}}\right]^{\frac{1}{2}} \mathrm{e}^{\frac{i}{\hbar} \Phi(q, \bar{q})}
$$

It can be shown to preserve the composition property and to be unitary within the semiclassical approximation.

The semiclassical approximation for $t_{n}$ involves the periodic manifolds of the classical map. For hyperbolic maps [18, 3],

$$
\left[t_{n}\right]_{\mathrm{scl}}=\sum_{p \in \mathcal{P}_{n}} \frac{n_{p} e^{i r\left(\Phi_{p} / \hbar-\nu_{p} \frac{\pi}{2}\right)}}{\left|\operatorname{det}\left(I-T_{p}^{r}\right)\right|^{\frac{1}{2}}} .
$$

The semiclassical approximation for $t_{n}$ involves the set of $n$-periodic orbits $\mathcal{P}_{n}$ which are repetitions of primitive periodic orbits of $\mathcal{F}$, with periods $n_{p}$ which are divisors of $n$, so that $n=n_{p} r$. The monodromy matrix is denoted by $T_{p}$. Each periodic orbit contribution is endowed with a phase which is the action summed along the periodic orbit,

$$
\Phi_{p}=\sum_{j=1}^{n_{p}} \Phi\left(q_{j}, q_{j+1}\right) \quad\left(\text { with } \quad q_{n_{p}+1}=q_{1}\right),
$$

and of the Maslov contribution $-\nu_{p} \pi / 2$.

For integrable maps, the action-angle variables $(I, \phi)$ will be used, where $I$ is the classical invariant. In the quantum picture, $I$ is quantized to integer multiples of $\hbar$ so that $I_{j}=j \hbar$ and $1 \leq j \leq M$. The matrix $U$ is diagonal in the action representation. The semiclassical approximation for the eigenphases can be carried out directly,

$$
\left[\theta_{j}\right]_{\mathrm{scl}}=\frac{1}{\hbar}\left[\Phi\left(f\left(I_{j}\right)\right)-I_{j} f\left(I_{j}\right)\right] .
$$

where $f(I)$ is the angular frequency (20). Thus,

$$
\left[t_{n}\right]_{\mathrm{scl}}=\sum_{j=1}^{M} \mathrm{e}^{i n\left[\theta_{j}\right]_{\mathrm{scl}}}
$$

This semiclassical expression is not of the desired form, because it does not express $t_{n}$ in terms of the periodic orbits. However, performing the $j$ sum using Poisson summation, one gets,

$$
\left[t_{n}\right]_{\mathrm{scl}}=\left(\frac{2 \pi}{n \hbar}\right)^{\frac{1}{2}} \mathrm{e}^{-i\left(n+\frac{1}{2}\right) \frac{\pi}{2}} \sum_{m=1}^{n}\left[\Phi^{\prime \prime}\left(\Delta \phi=2 \pi \frac{m}{n}\right)\right]^{\frac{1}{2}} \mathrm{e}^{\frac{i}{\hbar} n \Phi\left(\Delta \phi=2 \pi \frac{m}{n}\right)} .
$$

Now, $t_{n}$ is expressed as a sum over the periodic manifolds of period $n$ and winding number $m$. They occur at values of $I$ for which the angular frequency is rational $f\left(I_{n, m}\right)=2 \pi \frac{m}{n}$.

The expressions for $t_{n}$ in terms of periodic manifolds in the cases of classically integrable and classically chaotic maps are the necessary building blocks for the discussions which follows. 


\section{Compatibility of the semiclassical approximation}

The compatibility of the semiclassical approximation for the $t_{n}$ with the trace identities will be now shown for integrable maps. Turning first to (11), the semiclassical expression (26) for $t_{n}$ is substituted in the right hand side of (11) resulting in

$$
\begin{aligned}
& \epsilon \sum_{n=n_{0}}^{\infty}\left[t_{n}^{*} t_{n+\nu}\right]_{\mathrm{scl}} \mathrm{e}^{-n \epsilon}= \\
= & \epsilon \mathrm{e}^{-i \nu \frac{\pi}{2}} \frac{2 \pi}{\hbar} \sum_{n=n_{0}}^{\infty} \frac{\mathrm{e}^{-n \epsilon}}{(n(n+\nu))^{\frac{1}{2}}} \times \\
\times & \sum_{m=1}^{n} \sum_{m^{\prime}=1}^{n+\nu}\left(\Phi^{\prime \prime}\left(2 \pi \frac{m}{n}\right) \Phi^{\prime \prime}\left(2 \pi \frac{m^{\prime}}{n+\nu}\right)\right)^{\frac{1}{2}} \mathrm{e}^{\frac{i}{\hbar}\left[(n+\nu) \Phi\left(2 \pi \frac{m^{\prime}}{n+\nu}\right)-n \Phi\left(2 \pi \frac{m}{n}\right)\right]}
\end{aligned}
$$

The sum above runs over the periodic manifolds (tori) of the map. It is important to respect the integer character of $n, m, m^{\prime}$, since only for integer values, the classical orbits are periodic. If we turn these sums to integrals by e.g. Poisson summation, we would loose this feature. To get a finite contribution for (27) we must collect all the terms which contribute coherently to the sum. Summing over these terms (weighted by $\mathrm{e}^{-\epsilon n}$ ) should provide a pole at $\epsilon=0$, so that the final multiplication by $\epsilon$ will yield the residue at the pole. Inspecting (27) we immediately notice that, for example, the terms for which $\frac{m^{\prime}}{n+\nu}=\frac{m}{n}=\alpha(\nu)$ (where $\alpha(\nu)$ depends only on $\nu$ ) yield a contribution of the desired nature, because the net phase $\nu \Phi(2 \pi \alpha(\nu))$ is common to all the summed terms. To find these contributions in a consistent way, we shall identify them as the points where the first variation of the phase of the summand vanishes.

$$
\begin{aligned}
\delta\{\text { phase }\}=\delta n[ & \left(\Phi\left(2 \pi \frac{m^{\prime}}{n+\nu}\right)-\Phi\left(2 \pi \frac{m}{n}\right)\right) \\
& \left.-2 \pi\left(\frac{m^{\prime}}{n+\nu} \Phi^{\prime}\left(2 \pi \frac{m^{\prime}}{n+\nu}\right)-\frac{m}{n} \Phi^{\prime}\left(2 \pi \frac{m}{n}\right)\right)\right] \\
+\delta m^{\prime} & {\left[2 \pi \Phi^{\prime}\left(2 \pi \frac{m^{\prime}}{n+\nu}\right)\right]-\delta m\left[2 \pi \Phi^{\prime}\left(2 \pi \frac{m}{n}\right)\right] }
\end{aligned}
$$

The phase is stationary with respect to variations in $n$ when

$$
\frac{m^{\prime}}{n+\nu}=\frac{m}{n} \text {. }
$$

One should consider only the solutions in the range $m \leq n$ and $m^{\prime} \leq n+\nu$, consistently with the range of the sums over $m$ and $m^{\prime}$. The solution of (29) in integers is

$$
\begin{aligned}
& n=N \nu \quad \text { with } \quad N=N_{0}, \cdots, \infty \\
& m=N k \quad \text { with } \quad k=1, \cdots, \nu \\
& m^{\prime}=(N+1) k .
\end{aligned}
$$

The arbitrary integer $n_{0}$ is replaced by another arbitrary constant $N_{0}$ which fixes the lower limit of the $n$ sum. This solution is the general solution for the generic cases. One can always invent maps for which other stationary points exist. For each value of $k$, the points of stationary phase form a grid. Near each grid point, the summation variables will be replaced by local variables

$$
n=N \nu+\delta n ; m=N k+\delta m ; m^{\prime}=(N+1) k+\delta m^{\prime},
$$


with

$$
|\delta n| \leq \frac{\nu}{2} \quad ; \quad|\delta m| \leq \frac{k}{2} \quad ; \quad\left|\delta m^{\prime}\right| \leq \frac{k}{2} .
$$

The range of variation of the local variables is chosen such that each point in the original sum will be counted once. The contribution of the domain about each grid point will be computed by using the stationary phase approximation. The phase cannot be made stationary with respect to independent variations of $m$ and $m^{\prime}$ because there is no guarantee that $\Phi^{\prime}\left(2 \pi \frac{m^{\prime}}{n+\nu}\right)=0$ and $\Phi^{\prime}\left(2 \pi \frac{m}{n}\right)=0$ have solutions when $n$, $m$ and $m^{\prime}$ are integers. However, when (29) is satisfied, the phase is stationary on the manifold $\delta m=\delta m^{\prime}$. Thus, the sum over $n, m, m^{\prime}$ in (27) is replaced by

$$
\sum_{n=n_{0}}^{\infty} \sum_{m=1}^{n} \sum_{m^{\prime}=1}^{n+\nu} \rightarrow \sum_{k=1}^{\nu} \sum_{N=N_{0}}^{\infty}\left\{\sum_{\delta n=-\frac{\nu}{2}}^{\frac{\nu}{2}} \sum_{\delta m=-\frac{k}{2}}^{\frac{k}{2}} \sum_{\delta m^{\prime}=-\frac{k}{2}}^{\frac{k}{2}} \delta_{\delta m, \delta m^{\prime}}\right\}
$$

Where the curly brackets on the right enclose the contribution of the vicinity of a single grid point, restricted to the line $\delta m=\delta m^{\prime}$. On this line the summand is constant and therefore the summation amounts to multiplication by $k$. The $\delta n$ sum can be computed by considering the quadratic approximation to the phase near the stationary points and retaining the leading term in the result. It is determined by the curvature of the phase at the point where it is stationary

$$
\frac{\partial^{2}\{\text { phase }\}}{\partial n^{2}} \mid=-(2 \pi)^{2} \frac{k^{2}}{N(N+1) \nu^{3}} \Phi^{\prime \prime}\left(2 \pi \frac{k}{\nu}\right) .
$$

The amplitude of the result depends on $N$, but when it is substituted in (27) it exactly cancels the $N$ dependent coefficient. The phase of the $\delta n$ sum is $\nu \Phi\left(2 \pi \frac{k}{\nu}\right)$ which is also independent of $N$. Thus, the resulting terms of the $N$ sum depend on $N$ only through $\mathrm{e}^{-\epsilon \nu N}$. Summing and taking the limit $\epsilon \rightarrow 0$ results in a factor $\nu^{-1}$. Collecting all the factors, one finds that (27) is approximated by

$$
\begin{aligned}
& \lim _{\epsilon \rightarrow 0} \epsilon \sum_{n=n_{0}}^{\infty}\left[t_{n}^{*} t_{n+\nu}\right]_{\mathrm{scl}} \mathrm{e}^{-n \epsilon}= \\
& \left(\frac{2 \pi}{\nu \hbar}\right)^{\frac{1}{2}} \mathrm{e}^{-i\left(\nu+\frac{1}{2}\right) \frac{\pi}{2}} \sum_{k=1}^{\nu}\left[\Phi^{\prime \prime}\left(2 \pi \frac{k}{\nu}\right)\right]^{\frac{1}{2}} \mathrm{e}^{\frac{i}{\hbar} \nu \Phi\left(2 \pi \frac{k}{\nu}\right)}=\left[t_{\nu}\right]_{\mathrm{scl}} .
\end{aligned}
$$

This completes the proof that the trace identities (11) are compatible with the semiclassical approximation.

The more complex relations (17) or the equivalent (18) can be checked using the same technique. As an example, the compatibility of the identity

$$
\lim _{\epsilon_{1}, \epsilon_{2}=0} \epsilon_{1} \epsilon_{2} \sum_{n_{1}, n_{2}} t_{n_{1}+n_{2}}^{*} t_{n_{1}+\nu_{1}} t_{n_{2}+\nu_{2}} \mathrm{e}^{-n_{1} \epsilon_{1}-n_{2} \epsilon_{2}}=t_{\nu_{1}+\nu_{2}}
$$

will be demonstrated in some detail.

Substituting in (36) the semiclassical approximation (26) for $t_{n}$, one has to consider the sum

$$
\epsilon_{1} \epsilon_{2} \mathrm{e}^{-i\left(\nu_{1}+\nu_{2}\right) \frac{\pi}{2}}\left(\frac{2 \pi}{\hbar}\right)^{\frac{3}{2}} \sum_{n_{1}, n_{2}} \mathrm{e}^{-n_{1} \epsilon_{1}-n_{2} \epsilon_{2}}\left(\frac{1}{n_{1}+n_{2}} \frac{1}{n_{1}+\nu_{1}} \frac{1}{n_{2}+\nu_{2}}\right)^{\frac{1}{2}}
$$




$$
\begin{aligned}
& \times \sum_{m_{1}=1}^{n_{1}+\nu_{1}} \sum_{m_{2}=1}^{n_{2}+\nu_{2}} \sum_{m_{12}=1}^{n_{1}+n_{2}}\left[\Phi^{\prime \prime}\left(2 \pi \frac{m_{1}}{n_{1}+\nu_{1}}\right) \Phi^{\prime \prime}\left(2 \pi \frac{m_{2}}{n_{2}+\nu_{2}}\right) \Phi^{\prime \prime}\left(2 \pi \frac{m_{12}}{n_{1}+n_{2}}\right)\right]^{\frac{1}{2}} \\
& \times \mathrm{e}^{\frac{i}{\hbar}\left[\left(n_{1}+\nu_{1}\right) \Phi\left(2 \pi \frac{m_{1}}{n_{1}+\nu_{1}}\right)+\left(n_{2}+\nu_{2}\right) \Phi\left(2 \pi \frac{m_{2}}{n_{2}+\nu_{2}}\right)-\left(n_{1}+n_{2}\right) \Phi\left(2 \pi \frac{m_{12}}{n_{1}+n_{2}}\right)\right]}
\end{aligned}
$$

The phase of (37) can be made stationary with respect to variations of $n_{1}$ and $n_{2}$ under the following conditions:

$$
\begin{aligned}
& -\Phi_{12}+\Phi_{1}+\frac{m_{12}}{n_{1}+n_{2}} \Phi_{12}^{\prime}-\frac{m_{1}}{n_{1}+\nu_{1}} \Phi_{1}^{\prime}=0 \\
& -\Phi_{12}+\Phi_{2}+\frac{m_{12}}{n_{1}+n_{2}} \Phi_{12}^{\prime}-\frac{m_{2}}{n_{2}+\nu_{2}} \Phi_{2}^{\prime}=0
\end{aligned}
$$

Where the short-hand notation $\Phi_{1}=\Phi\left(2 \pi \frac{m_{1}}{n_{1}+\nu_{1}}\right)$ and $\Phi_{1}^{\prime}=2 \pi \Phi^{\prime}\left(2 \pi \frac{m_{1}}{n_{1}+\nu_{1}}\right)$, etc was used. The conditions (38) are satisfied by solutions in integers of:

$$
\frac{m_{12}}{n_{1}+n_{2}}=\frac{m_{1}}{n_{1}+\nu_{1}} \leq 1 \text { and } \frac{m_{12}}{n_{1}+n_{2}}=\frac{m_{2}}{n_{2}+\nu_{2}} \leq 1 .
$$

The general solutions depend on three integers $N_{1}, N_{2}$ and $k$ so that

$$
\begin{aligned}
& n_{1}=\left(N_{1}-1\right) \nu_{1}+N_{1} \nu_{2} \text { with } N_{1}=N_{0}, \cdots, \infty \\
& n_{2}=N_{2} \nu_{1}+\left(N_{2}-1\right) \nu_{2} \text { with } N_{2}=N_{0}, \cdots, \infty \\
& m_{12}=\left(N_{1}+N_{2}-1\right) k \quad \text { with } 1 \leq k \leq \nu_{1}+\nu_{2} \\
& m_{1}=N_{1} k \\
& m_{2}=N_{2} k
\end{aligned}
$$

Again, for each value of $k$ the points of stationary phase form a grid and one computes seperately the contribution from the vicinity of each grid point. The summation volume about each grid point is of size

$$
\left|\delta n_{1}, \delta n_{2}\right| \leq \frac{\nu_{1}+\nu_{2}}{2} ;\left|\delta m_{1}, \delta m_{2}\right| \leq \frac{k}{2} \quad ; \quad\left|\delta m_{12}\right| \leq k
$$

The phase cannot be made stationary with respect to independent variations of $m_{1}, m_{2}$ and $m_{12}$. However, like in the previous case, the phase is constant for

$$
\delta m_{12}=\delta m_{1}+\delta m_{2}
$$

The sums in (37) are rewritten in terms of the local variables for each grid point, and the curly brackets encloses the sums over individual domains (41).

$$
\begin{aligned}
& \sum_{n_{1}=n_{0}}^{\infty} \sum_{n_{2}=n_{0}}^{\infty} \sum_{m_{1}=1}^{n_{1}+\nu_{1}} \sum_{m_{2}=1}^{n_{2}+\nu_{2}} \sum_{m_{12}=1}^{n_{1}+n_{2}} \rightarrow \\
& \sum_{k=1}^{\nu_{1}+\nu_{2}} \sum_{N_{1}=N_{0}}^{\infty} \sum_{N_{2}=N_{0}}^{\infty}\left\{\sum_{\delta n_{1}} \sum_{\delta n_{2}} \sum_{\delta m_{1}} \sum_{\delta m_{2}} \delta_{\delta m_{12}, \delta m_{1}+\delta m_{2}}\right\}
\end{aligned}
$$

The last Kronecker $\delta$ is due to the restriction (42). Since the summands in the $\delta m_{1}, \delta m_{2}$ sums are constant, they contribute a factor of $k^{2}$. The $\delta n_{1}, \delta n_{2}$ sum is performed again by expanding the phase to second order, and retaining the leading term in the result. The determinant of second derivatives at the point of stationary 
phase is

$$
\begin{aligned}
& \operatorname{det}\left(\frac{\partial^{2}\{\text { phase }\}}{\partial n_{i} \partial n_{j}}\right)= \\
& -\left(2 \pi \frac{k}{\nu_{1}+\nu_{2}}\right)^{4}\left(\Phi^{\prime \prime}\left(2 \pi \frac{k}{\nu_{1}+\nu_{2}}\right)\right)^{2} \frac{\nu_{1}+\nu_{2}}{\left(n_{1}+n_{2}\right)\left(n_{1}+\nu_{1}\right)\left(n_{2}+\nu_{2}\right)},
\end{aligned}
$$

where $n_{1}$ and $n_{2}$ take the values (40). Collecting all the factors, and performing the $N_{1}$ and $N_{2}$ sums while taking the limit $\epsilon_{1}, \epsilon_{2} \rightarrow 0$, one remains with the sum over $k$ which can be easily identified as $\left[t_{\nu_{1}+\nu_{2}}\right]_{\mathrm{scl}}$.

This method can be extended to identities involving higher powers. The procedure becomes much more cumbersome and will not be reproduced here.

The essence of the derivations outlined above is that the phase of the summands is constant on an infinite grid of integers. Only when all of them are summed together, they provide the necessary singularity which is cancelled against the $\epsilon$ factors and give the correct answer.

The compatibility of the trace identities with the semiclassical approximation demonstrate the importance of "non-diagonal" correlations which are essentially due to the repetitive nature of the distribution of periodic orbits for integrable maps. As a matter of fact, had one applied the standard diagonal approximation where repetitions are neglected, to the sums (27),(37) one would get a vanishing result when $\nu$ or $\left(\nu_{1}+\nu_{2}\right) \neq 0$. The condition (29) picks up (non-diagonal) pairs of $n$-periodic and $n+\nu$ periodic manifolds which coincide, since the action variable $I_{n, m}$ is the same. This feature is effective in integrable systems since the periodic manifolds are specified completely by integers (the period $n$ and the winding number $m$ ), and it is responsible for the compatibility of the trace identity with the semiclassical approximation. The condition (40) expresses a similar coincidence of three periodic manifolds. In chaotic systems, repetitions do exist but they play a much less important rôle, because apart from the period $n$ there exists no other integer which would replace $m$ to specify the periodic orbits. This is why the methods described in the present section fails for the chaotic case. The substitution of the expressions (22) for systems which are chaotic in the classical limit, and attempts to identify the classical correlations which are implied by the trace identities failed, so far, to give a definite answer.

\section{Discussion}

In the previous section, the compatibility of the trace identities with the semiclassical quantization of integrable maps was studied from the point of view of periodic orbit theory. It can be also proven very simply by observing that the semiclassical expressions (25) for the $t_{n}$ has the formal structure as a trace of a unitary matrix, and therefore the trace identities which are based on nothing else but on this form, are automatically ensured. This result does not detract from the work presented in the previous section, because it gave new insight into the interplay between the trace identities and the correlations in the spectrum of classical actions.

In general, the semiclassical approximation for a quantum evolution operator is the leading approximant in an asymptotic expansion, in which the time is kept fixed and $\hbar$ approaches zero. Taking the opposite order in which $\hbar$ is fixed and the time is increased, is not allowed within the semiclassical approximation. One has to bear this basic rule in mind when one tests the semiclassical approximation. In the present 
context, the magnitude of $\epsilon^{-1}$ sets the scale of the relevant evolution time (see (13)). Hence the proper order of limits would seem to be such that $\epsilon$ be fixed while $\hbar \rightarrow 0$. However, the semiclassical limit is synonymous to $M \rightarrow \infty$ (2). In this limit the spectrum of $U$ becomes dense on the unit circle, and the trace identities do not hold. These conflicting demands can be satisfied when $\epsilon \leq \mathcal{O}\left(\hbar^{f}\right)$. This remark does not apply to the test we performed for integrable maps, because the formal structure of $\left[t_{n}\right]_{\mathrm{scl}}$ as given by (25) is compatible with the trace identities for any values of $\hbar$ and $n$. This is not the case for quantum maps which correspond to classically chaotic systems.

So far, any attempt to assess the compatibility of the trace identities with the semiclassical approximation for classically chaotic systems ended in failure. Even for maps on graphs, where the periodic orbit expansion of $t_{n}$ is exact [19, 20, 21, I was unable to derive the trace identities within periodic orbit theory. Therefore, the nature of the underlying classical correlations which ensure the exact trace identities when they are expressed in terms of periodic orbits remains an enigma, and calls for further research. In Appendix A, the trace identities are used to derive some combinatorial identities, which illustrates an application outside of the domain of periodic orbits theory.

\section{Acknowledgement}

The author thanks the Humboldt foundation for the award which enabled him to perform this research at the Philipps-Universitaet Marburg. The important rôle of Professor Eugene Bogomolny to this work was mentioned in the text, and it is a pleasure to thank him again. I am indebted to Dr Harel Primack for his critique and for the helpful comments and suggestions. Appendix A follows from the work on quantum graphs and combinatorics which is conducted in collaboration with Dr Holger Schanz, whose cooperation is acknowledged. I am indebted to Professor Bruno Eckhardt for the hospitality in Marburg and for useful discussions. The work was supported in part by the Minerva Center for Nonlinear Physics of Complex Systems, and by a grant from the Israel Science Foundation.

\section{Appendix A. Identities for Krawtchouk polynomials}

We consider here the quantum map for a 2-star graph which was defined and studied in detail in 21]. It consists of a "central" vertex out of which emerge 2 bonds, terminating at vertices (with indices $j=1,2$ ) with valencies $v_{j}=1$. The ratio between the bond lengths $L_{j}$ is assumed to be irrational. This simple model is not completely trivial if the central vertex scattering matrix is chosen as

$$
\sigma^{(o)}=\left(\begin{array}{ll}
\frac{1}{\sqrt{2}} & \frac{\mathrm{i}}{\sqrt{2}} \\
\frac{\mathrm{i}}{\sqrt{2}} & \frac{1}{\sqrt{2}}
\end{array}\right) .
$$

Neumann boundary conditions are imposed at the two other vertices. The Hilbert space is of dimension 2 and the evolution operator is

$$
U(k)=\left(\begin{array}{ll}
\mathrm{e}^{2 i k L_{1}} & 0 \\
0 & \mathrm{e}^{2 i k L_{2}}
\end{array}\right)\left(\begin{array}{ll}
\frac{1}{\sqrt{2}} & \frac{\mathrm{i}}{\sqrt{2}} \\
\frac{\mathrm{i}}{\sqrt{2}} & \frac{1}{\sqrt{2}}
\end{array}\right) .
$$


where the diagonal matrix on the left takes care of the free propagation on the bonds and the reflections from the vertices $j=1,2$.

One can write an exact expression for $t_{n}(k)=\operatorname{tr} U^{n}(k)$ in terms of periodic orbits on the 2-star graph which is analogous to (22). There are $2^{n} / n n$-periodic orbits. However, their lengths can take only $n+1$ distinct values : $L(n, q)=2\left(q L_{1}+(n-q) L_{2}\right)$, with $0 \leq q \leq n$. Thus, one can write

$$
t_{n}(k)=\sum_{q=0}^{n} \mathrm{e}^{i L(n, q) k} A(n, q),
$$

where $A(n, q)$ is the coherent sum of the amplitudes contributed by the isometric orbits with length $L(n, q)$. It can be computed explicitly [21] in terms of Krawtchouk Polynomials

$$
A(n, q)=\frac{1}{\sqrt{2^{n}}} \begin{cases}1 & \text { for } \quad q=0 \text { or } n \\
(-1)^{n+q} \sqrt{\frac{n}{q}\left(\begin{array}{l}
n \\
q
\end{array}\right)} P_{n-1, n-q}(q) & \text { for } \quad 0<q<n .\end{cases}
$$

and the Krawtchouk polynomials are defined as in [13, 14] by

$$
P_{N, k}(x)=\left(\begin{array}{c}
N \\
k
\end{array}\right)^{-1 / 2} \sum_{\nu=0}^{k}(-1)^{k-\nu}\left(\begin{array}{l}
x \\
\nu
\end{array}\right)\left(\begin{array}{c}
N-x \\
k-\nu
\end{array}\right) \text { for } 0 \leq k \leq N .
$$

Substituting (A3) in the trace identity (11), we get for any integers $\nu$ and $n_{0}$

$$
\begin{aligned}
\lim _{\epsilon \rightarrow 0} \epsilon & \sum_{n=n_{0}}^{\infty} \mathrm{e}^{-n \epsilon} \sum_{q=0}^{n} \sum_{p=0}^{n+\nu} \mathrm{e}^{2 i k\left[(p-q) L_{1}+(\nu-(p-q)) L_{2}\right]} A(n+\nu, p) A(n, q) \\
& =\sum_{\kappa=0}^{\nu} A(\nu, \kappa) \mathrm{e}^{2 i k\left[\kappa L_{1}+(\nu-\kappa) L_{2}\right]}
\end{aligned}
$$

This is valid for any value of the wave number $k$. When the lengths $L_{1}$ and $L_{2}$ are incommensurate, the equality can hold only if the coefficients of the phase factors $\mathrm{e}^{2 i k L(\nu, \kappa)}$ on both sides are equal. This implies

$$
\lim _{\epsilon \rightarrow 0} \epsilon \sum_{n=n_{0}}^{\infty} \mathrm{e}^{-n \epsilon} \sum_{q=0}^{n} A(n+\nu, q+\kappa) A(n, q)=A(\nu, \kappa),
$$

with $0 \leq \kappa \leq \nu$. Restricting to $\kappa$ to the interval $1 \leq \kappa \leq \nu-1$ the identity can be expressed in terms of Krawtchouk polynomials exclusively.

$$
\begin{aligned}
& \sqrt{\frac{\nu}{\kappa}\left(\begin{array}{c}
\nu \\
\kappa
\end{array}\right)} P_{\nu-1, \nu-\kappa}(\kappa)=\lim _{\epsilon \rightarrow 0} \epsilon \sum_{n=n_{0}}^{\infty} \frac{\mathrm{e}^{-n \epsilon}}{2^{n}} \times \\
\times & \sum_{q=0}^{n} \sqrt{\frac{n(n+\nu)}{q(q+\kappa)}\left(\begin{array}{c}
n \\
q
\end{array}\right)\left(\begin{array}{c}
n+\nu \\
q+\kappa
\end{array}\right)} P_{n-1, n-q}(q) P_{n+\nu-1, n+\nu-q-\kappa}(q+\kappa) .
\end{aligned}
$$

I could not find identities of this kind in the standard books on Krawtchouk polynomials. 


\section{References}

[1] E.B. Bogomolny Semiclassical quantization of multidimensional systems. Nonlinearity 5 (1992) 805.

[2] E. Doron and U. Smilansky. Semiclassical Quantization of Chaotic Billiards - a Scattering Theory Approach. Nonlinearity 5 (1992) 1055.

[3] U. Smilansky. Semiclassical Quantization of Chaotic Billiards - A Scattering Approach. in Proc. of the Les Houches Summer School on Mesoscopic Quantum Physics. Elsevier Science Publ. (1995) Ed. E. Akkermans, G. Montambaux and J. L. Pichard.

[4] F. Haake, M. Kuś, H.-J. Sommers, H. Schomerus, and K. Zyczkowski. Secular determinants of random unitary matrices. J. Phys. A 29 (1996) 3641.

[5] B. Eckhardt and F. Haake. Periodic orbit quantization of Baker's map. J. Phys. A 27 (1994) 4449-4455.

[6] M.V. Berry. Semiclassical Theory of Spectral Rigidity. Proc. Royal Soc. Lond A 400 (1985) 229.

[7] J. Keating The semiclassical sum rule and Riemann's zeta function, in Quantum Chaos, eds Cerdeira, Ramaswamy, Gutzwiller and Casati (World Scientific 1991), 280-294.

[8] R. Connors PhD Thesis, Bristol University (1998).

[9] N. Argaman, F. Dittes , E. Doron, J. Keating, A. Kitaev, M. Sieber and U. Smilansky. Correlations in the actions of Periodic Orbits Derived from Quantum Chaos. Phys. Rev. Letters 71, (1993) 4326-4329

[10] F.M. Dittes, E. Doron and U. Smilansky. Long time Behavior of the Semiclassical Baker's Map Phys Rev E 49,(1994) R963-R966

[11] D. Cohen, H. Primack and U. Smilansky. Quantal- classical duality and the semiclassical trace formula. Ann. of Phys.264 (1998), 108-170.

[12] E. Bogomolny (private communication)

[13] G. Szegö, Orthogonal polynomials, American Mathematical Society Colloquium Publications, Vol. 23, New York (1959).

[14] A. F. Nikiforov, S. K. Suslov, and V. B. Uvarov, Classical Orthogonal Polynomials of a Discrete Variable, Springer Series in Computational Physics, Berlin (1991).

[15] G. Cohen, I. Honkala, S. Litsyn, and A. Lobstein, Covering Codes, North Holland Mathematical Library, Vol. 54, (1997).

[16] F.J. Dyson. Statistical Theory of the Energy Levels of Complex Systems J. Math. Phys. 3 (1962) 140.

[17] W. H. Miller. Classical-limit quantum mechanics and the theory of molecular collisions Adv. Chem. Phys. 25(1974) 69.

[18] M. Tabor. A Semiclassical Quantization of Area-Preserving Maps. Physica D6, 195 (1983)

[19] T. Kottos and U. Smilansky. Quantum Chaos on Graphs Phys. Rev. Lett. 79 (1997), 4794.

[20] T. Kottos and U. Smilansky. Periodic Orbit Theory and Spectral Statistics for Quantum Graphs Annals of Physics 273, 1 (1999).

[21] H. Schanz and U. Smilansky. Spectral Statistics for Quantum Graphs: Periodic Orbits and Combinatorics Proceedings of the Australian Summer School on Quantum Chaos and Mesoscopics ,Canberra, Australia in press (1999). 